\title{
The scaling of human basal and resting metabolic rates
}

\author{
Heather M. Bowes ${ }^{1}$ - Catriona A. Burdon ${ }^{1}$ - Nigel A. S. Taylor ${ }^{1}$ (C)
}

Received: 21 May 2020 / Accepted: 23 September 2020

(c) Springer-Verlag GmbH Germany, part of Springer Nature 2020

\begin{abstract}
Purpose In tachymetabolic species, metabolic rate increases disproportionately with body mass, and that inter-specific relationship is typically modelled allometrically. However, intra-specific analyses are less common, particularly for healthy humans, so the possibility that human metabolism would also scale allometrically was investigated.

Methods Basal metabolic rate was determined (respirometry) for 68 males (18-40 years; $56.0-117.1 \mathrm{~kg}$ ), recruited across five body-mass classes. Data were collected during supine, normothermic rest from well-rested, well-hydrated and postabsorptive participants. Linear and allometric regressions were applied, and three scaling methods were assessed. Data from an historical database were also analysed (2.7-108.9 kg, 4811 males; $2.0-96.4 \mathrm{~kg}, 2364$ females).

Results Both linear and allometric functions satisfied the statistical requirements, but not the biological pre-requisite of an origin intercept. Mass-independent basal metabolic data beyond the experimental mass range were not achieved using linear regression, which yielded biologically impossible predictions as body mass approached zero. Conversely, allometric regression provided a biologically valid, powerful and statistically significant model: metabolic rate $=0.739 *$ body mass ${ }^{0.547}$ $(P<0.05)$. Allometric analysis of the historical male data yielded an equivalent, and similarly powerful model: metabolic rate $=0.873 *$ body mass ${ }^{0.497}(P<0.05)$.

Conclusion It was established that basal and resting metabolic rates scale allometrically with body mass in humans from $10-117 \mathrm{~kg}$, with an exponent of $0.50-0.55$. It was also demonstrated that ratiometric scaling yielded invalid metabolic predictions, even within the relatively narrow experimental mass range. Those outcomes have significant physiological implications, with applications to exercising states, modelling, nutrition and metabolism-dependent pharmacological prescriptions.
\end{abstract}

Keywords Allometry $\cdot$ Basal metabolic rate $\cdot$ Body mass $\cdot$ Metabolic rate $\cdot$ Oxygen consumption $\cdot$ Scaling $\cdot$ Size

\section{Abbreviations}

AIC Akaike information criterion

CI Confidence interval

$\mathrm{CV} \quad$ Coefficient of variance

$r^{2} \quad$ Coefficient of determination

RMSE Root-mean-square error

SD Standard deviation

Communicated by Jean-René Lacour.

Nigel A. S. Taylor

nigelastaylor@gmail.com

1 Centre for Medical and Exercise Physiology, School of Medicine, University of Wollongong, Wollongong, NSW 2522, Australia

\section{Introduction}

Intra- and inter-specific (species) comparisons of physiological and morphological variables often require data scaling to help elucidate mechanistic relationships between, or to remove the influence of body size on, the dependent variables of interest. In this communication, the emphasis is on the scaling of the basal and resting metabolic rates of healthy humans, for which a wide variability is observed across the adult population (Harris and Benedict 1919; Quenouille et al. 1951; Schofield 1985; Cole and Henry 2005). About $70-80 \%$ of that variance is attributable to differences in body mass (Elia 1992; Johnstone et al. 2005; Müller et al. 2013), as the possession of more cells comes with obligatory energy demands.

In the animal kingdom, there exists theoretical (Brody 1945; Heusner 1982a; Schmidt-Nielsen 1984; Darveau et al. 2002; White and Kearney 2014) and empirical evidence (Sarrus and Rameaux 1838; Kleiber 1932; White 
and Seymour 2005) which leaves little doubt that resting metabolic rates scale allometrically with body mass. However, the bulk of that modelling was performed using interspecific data obtained from species varying in body mass over many orders of magnitude (Kleiber 1932; White and Seymour 2005; Sieg et al. 2009; White et al. 2012), and resulting in an allometric scaling exponent of 0.67 (metabolic rate $=$ constant $*$ body mass ${ }^{0.67}$; White and Seymour 2005). Intra-specific allometric analyses, on the other hand, cover considerably smaller mass ranges, are much less common (Rubner 1883 [dogs]; Refinetti 1989 [rats]; Kvist and Lindström 2001 [birds]) and have rarely included humans. Instead, human data are more frequently modelled using other scaling approaches (Harris and Benedict 1919; Boothby and Sandiford 1922; Durnin 1959; Schofield 1985).

Several groups have used an allometric (power regression) approach to scale oxygen consumption in humans during rest, as well as during steady-state and maximal exercise (Nevill et al. 1992; Rogers et al. 1995; Batterham and Jackson 2003). To the best of our knowledge, however, such analyses have not been applied to homogeneous human samples, nor have participants been studied under basal conditions. Furthermore, within the exercise domain, linear regression has traditionally been used to scale oxygen consumption (Jetté et al. 1990; Hall et al. 2004), with data frequently being reported as a ratio standard. Linear scaling has also been applied to basal metabolic data (Harris and Benedict 1919; Schofield 1985; Cole and Henry, 2005). Such approaches may reflect a reluctance to apply more rigorous analyses, or they may simply mean that, over the relatively small mass ranges investigated, those relationships appeared linear. Nonetheless, it has resulted in linearly scaled, metabolic and oxygen consumption descriptions dominating the human literature, even though the appropriateness of such scaling has been vigorously questioned for more than a century (Krogh 1916; Sholl 1948; Tanner 1949; Gould 1966; Packard and Boardman 1999).

Therefore, the aim of this experiment was to revisit metabolic scaling in humans, and, in so doing, to explore the possibility that, even within the narrow adult body-mass range, basal metabolism might also scale allometrically. The intention was not to investigate the mechanisms through which metabolic rate is linked with body mass. Instead, the emphasis was on the elimination of that effect, so that healthy individuals of different body masses (metabolic sizes; Kleiber 1961) might be meaningfully compared. This was firstly examined under conditions where oxygen supply meets metabolic demand (basal and resting states). Eventually, that would be expanded to encompass the complete metabolic range (aerobic scope) using steady-state ambulation (with and without load carriage) through to maximal exercise (unloaded running), during which demand often exceeds supply. The ultimate objective was to increase our understanding of the metabolic impact that load carriage has on adults of varying body mass.

Herein, the first of a series of studies is communicated. This investigation involved the scaling of basal metabolic rate in a homogeneous, experimental sample of adults. Then, in combination with an historical database, scaling was performed across body masses varying by two orders of magnitude. In each case, data were scaled using both linear and allometric (non-linear) approaches. It was hypothesised that human basal metabolic data would scale allometrically, with that method providing the most appropriate normalisation method through which the impact of mass-dependent variations in metabolism might be removed.

\section{Methods}

\section{Participants}

Three healthy, population samples were used in this project; one adult (male) sample was recruited from University students (the experimental participants), and metabolic data from separate male and female samples were extracted from the literature (historical database participants: Schofield 1985). Every experimental subject provided written, informed consent prior to participation. All procedures were approved by a Human Research Ethics Committee (University of Wollongong, Australia: HE14/469) in accordance with national regulations (National Health and Medical Research Council), and in compliance with the Declaration of Helsinki.

\section{Experimental participants}

Intra-specific investigations of metabolic scaling require data to be collected from the largest possible body-mass range. For humans, that has been achieved for resting states by investigating children and adults of both genders (Boothby and Sandiford 1922; Schofield 1985; Rogers et al. 1995). However, since inter-individual variability influences experimental power independently of the sample size (White and Seymour 2005), then recruitment criteria become critical design considerations. In the first instance, the impact of sexual dimorphism was eliminated using a single-gender sample for the experimental participants. Secondly, to eliminate disproportionate and aged-dependent variations in the masses of the most metabolically active organs (Holliday et al. 1967; Holliday 1971; Müller et al. 2011), children and adolescents ( $<18$ years), as well as older adults ( $>40$ years), were excluded, resulting in a generally homomorphic experimental sample (Heusner 1982b). For the Australian population, those recruitment restrictions resulted in a probable 
mass range from 55-110 kg (5th-95th percentiles: Australian Bureau of Statistics 2012).

Participants were recruited with approximately equal numbers within each of five body-mass classifications (extra small, 55-65 kg; small, 66-76 kg; medium, 77-87 kg; large, 88-98 kg; extra large, $>99 \mathrm{~kg}$ ), each of which was separated by a $1-\mathrm{kg}$ interval. That approach minimised bias that can occur when distributions favour either end of the mass spectrum (White and Seymour 2005). An a priori power test showed that 50-60 participants were required for the linear modelling of metabolic data (Green 1991). Exploratory analyses were performed using an arbitrary exponent (mass ${ }^{0.67}$ ), with bootstrapping used to predict means and standard errors of those means for sub-group sample sizes of 5-30, in increments of five. Sample sizes of 10-15 individuals halved the predicted standard error of the exponent, relative to a sample of five, but adding more subjects had minimal further impact. Therefore, the sample size for each mass classification was set at 10-15 (i.e., 50-75 individuals overall).

Sixty-eight healthy, physically-active men, who engaged in endurance exercise at least three times per week, completed this investigation (18-34 years; Table 1). Each was screened to eliminate cardiovascular, respiratory or musculoskeletal pathologies that might affect subsequent load carriage trials. To standardise body-fat content and to minimise body-size bias, subcutaneous adiposity was estimated and normalised to a stature of $170.18 \mathrm{~cm}$ (Ross and Wilson 1974). That method was used because individuals with similar subcutaneous adiposity, but of varying stature, will have a different relative adiposity (Ross and Marfell-Jones 1991). Moreover, since the adipose-free body mass accounts for $\sim 80 \%$ of the resting metabolic rate (Müller et al. 2018), then variations in body composition would increase the interindividual variance in basal metabolic rate (Holliday et al. 1967; Holliday 1971; Müller et al. 2013). Therefore, recruitment was also based on subjects having an average-to-low subcutaneous adiposity, as defined by the height-adjusted sum of six skinfold thicknesses being $\leq 88 \mathrm{~mm}$. Six of the 68 participants exceeded that threshold, but only three were potential outliers. When closely evaluated (Analysis of Covariance), those individuals did not significantly influence either the proposed linear or allometric modelling outcomes $(P>0.05)$, so their data were retained.

\section{Historical participants}

Resting metabolic rates for two additional (independent) population samples were extracted from the literature (one male and one female dataset). Arguably the most extensive, and perhaps also the most carefully filtered compilations of such data are contained within Schofield (1985; Appendix 3). Those data were extracted from literature published over the preceding 60 years, although the level of experimental control would vary across time and laboratories. The reasons for using those datasets were two-fold. In the first instance, a cross-validation of the scaling model derived using the experimental participants would be undertaken by applying that model to data from an independent and very large (male) population sample. That sample included 4811 males, aged between 0.02 and 52.3 years, and covered a body-mass range from $2.7-108.9 \mathrm{~kg}$. A second historical sample was also used, which contained 2364 females (body mass, $2.0-96.4 \mathrm{~kg}$; age, 0.14-64.0 years). Now the objective was to evaluate the gender-independence of scaling models obtained using the male data. For both datasets, morphological and resting metabolic means (with standard deviations and sample sizes) were provided in increments of $1 \mathrm{~kg}$ (bins), with 101 and 93 body-mass classifications (class intervals), respectively.

In all such data-retrieval exercises, just as there is with meta-analyses, there is a reliance upon the skill and precision of the original investigators, and the validity of those data. Schofield (1985) was particularly careful with regard

Table 1 Physical characteristics of the experimental participants

\begin{tabular}{llllllll}
\hline $\begin{array}{l}\text { Participant size } \\
\text { classifications }\end{array}$ & Sample sizes & Age (years) & Height $(\mathrm{cm})$ & Body mass $(\mathrm{kg})$ & $\begin{array}{l}\text { Height-adjusted } \\
\text { body mass }(\mathrm{mm})\end{array}$ & $\begin{array}{l}\text { Sum of skinfolds (mm) } \\
\text { Height-adjusted } \\
\text { adiposity (mm) }\end{array}$ \\
\hline All participants & 68 & $22.5(3.1)$ & $181.6(8.7)$ & $80.9(14.7)$ & $66.2(7.7)$ & $61.6(28.6)$ & $56.6(25.3)$ \\
Ranges & & $18-34$ & $163.0-201.5$ & $56.0-117.1$ & $50.2-91.0$ & $28.0-158.0$ & $28.5-151.5$ \\
Extra small & 12 & $21.8(3.2)$ & $172.7(5.2)$ & $61.3(2.2)$ & $58.7(5.6)$ & $42.1(10.5)$ & $41.6(10.8)$ \\
Small & 16 & $21.6(2.1)$ & $174.5(4.5)$ & $71.5(3.1)$ & $66.4(4.8)$ & $55.6(18.6)$ & $51.8(16.6)$ \\
Medium & 19 & $22.6(3.8)$ & $184.1(3.5)$ & $81.4(2.4)$ & $64.4(5.0)$ & $54.3(16.9)$ & $50.4(16.2)$ \\
Large & 13 & $23.6(2.6)$ & $187.6(6.2)$ & $93.8(2.7)$ & $70.4(7.7)$ & $67.8(20.4)$ & $61.7(19.3)$ \\
Extra large & 8 & $23.3(3.7)$ & $193.0(8.2)$ & $107.4(5.1)$ & $74.2(9.8)$ & $109.4(43.2)$ & $94.4(41.9)$
\end{tabular}

Data are presented as means with standard deviations in parentheses, and ranges

The height-adjusted measures are anthropological data adjusted to a stature of $170.18 \mathrm{~cm}$ (Ross and Wilson 1974), as described in the "Methods". Skinfold thicknesses are the sum of values measured at six sites (see "Methods") 
to data rejection, and only included data "judged to be scientifically sound and which provided complete reports on the relevant data" (P 11). Nevertheless, some noise was inevitable and, although those data were reported as basal, the definition applied herein would classify those data as resting metabolic rates.

\section{Procedures}

\section{Experimental overview}

On presentation, the experimental participants changed into standardised clothing (pre-fitted long trousers, longsleeved shirt and thick socks) and consumed $150 \mathrm{~mL}$ of warm water $\left(37^{\circ} \mathrm{C}\right)$, whereupon a deep-body temperature sensor, a heart rate monitor and an oronasal mask were fitted. Subjects adopted a supine position on a firm bed and pillow for $60 \mathrm{~min}$. When comfortable, a thin blanket was positioned over the abdomen, legs and feet. Participants were instructed to remain stationary, but awake, and were supervised throughout to ensure adherence.

\section{Experimental standardisation}

Rigid pre-experimental procedures were implemented, and universally followed, thus ensuring that energy exchanges between each subject and the environment were minimal, and that truly basal conditions were established. Participants presented in a post-absorptive state, they were well rested and normally hydrated, and data were collected under normothermic conditions. This required a $12-\mathrm{h}$, overnight fast that followed the consumption of a high-carbohydrate, lowfat evening meal, but with normal (water-only) fluid intakes over that duration. Subjects avoided strenuous physical activity within the previous $24 \mathrm{~h}$, then presented at 07:00 $\mathrm{h}$ following adequate sleep $(\sim 8 \mathrm{~h})$. Testing was conducted within a locked, air-conditioned laboratory $\left(\sim 23{ }^{\circ} \mathrm{C} ; \sim 50 \%\right.$ relative humidity) with low-level lighting and minimal noise. Laboratory through traffic was prevented. The clothing and blanket ensured the attainment of a consistent thermal state across participants, as confirmed from deep-body temperatures. Finally, external stimuli were minimised, with subjects wearing an eye mask and ear protectors.

\section{Measurements on the experimental sample}

Anthropometric measures and six skinfold thicknesses were recorded (triceps, subscapular, supraspinale, abdominal, anterior thigh and medial calf: Eiken skinfold calliper, Meikosha, Tokyo, Japan) and summed. Two measurements from each site were averaged, unless differing by $>5 \mathrm{~mm}$, in which case a third measurement was performed, with the mode then used. Body masses (MS3200, Medical Scale,
Charder, Taichung, Taiwan) and the summed skinfold data were then height adjusted using the method of Ross and Wilson (1974): mass $[\mathrm{kg}] *(170.18 / \text { height }[\mathrm{cm}])^{3}$; and skinfold sum $[\mathrm{cm}] * 170.18 /$ height $[\mathrm{cm}]$. Those indices formed part of the subject-selection criteria.

Basal oxygen consumption (open-circuit respirometry), heart rate and deep-body temperature (insulated auditory canal) were measured continuously (60 min). The implicit assumption of respirometry was that the steady-state ratio of carbon dioxide produced to oxygen consumed can be approximated using data collected at the mouth (respiratory exchange ratio), and that it provides a valid reflection of cellular gas exchanges, the respiratory quotient and metabolic rate (Shephard 2017; Taylor et al. 2018). Standardisation procedures were aimed at satisfying the conditions necessary for those assumptions to be valid. To minimise the impact of respiratory artefacts, a correctly fitted oronasal mask was worn for $60 \mathrm{~min}$, with oxygen consumption recorded throughout. Data from the first 15 min were discarded, as were values from the last $5 \mathrm{~min}$, and at any time when a participant moved. Those data reflected nonbasal states. Thus, data-collection periods were $\sim 40 \mathrm{~min}$. The mean oxygen consumption over that period was converted to a metabolic rate (Weir 1949), with the thermal equivalent of oxygen for the non-protein respiratory quotient determined on an individual basis. That procedure has an error of $<1 \%$ under basal conditions (Mansell and MacDonald 1990). The resulting data represented basal metabolism (megajoules [MJ]), and are reported as daily metabolic rates $\left(\mathrm{MJ} \mathrm{day}^{-1}\right)$.

Expired gas fractions and expiratory flows were sampled from a two-way valve (7400 series, Hans Rudolph, Kansas, USA), and analysed continuously (TrueOne 2400, ParvoMedics Inc. Utah, USA). Those data were used to derive oxygen consumption, carbon dioxide production and minute ventilation as 15 -s averages. Two-point gas (room air plus alpha standard gases [16.00\% oxygen, $4.00 \%$ carbon dioxide, $80 \%$ nitrogen]) and a range of flow calibrations (50 to $>300 \mathrm{~L} \mathrm{~min}^{-1}$ ) preceded data collection. Heart rate was monitored from ventricular depolarisation (15-s intervals; Polar Electro, Kempele, Finland).

Since metabolic reactions are temperature dependent, deep-body temperature was measured so that normothermia could be verified and sustained. Measurements were taken from the auditory canal using a thermistor embedded into a moulded plug, and protruding $1 \mathrm{~cm}$ (Edale instruments Ltd. Cambridge, UK). The ear was insulated to minimise the affects of a cooler laboratory, and that method provides a dynamically responsive index of oesophageal (Todd et al. 2014) and intra-thoracic blood temperatures (Taylor et al. 2014). Data were sampled at 15 -s intervals (Grant Instruments Ltd. 1206 Series Squirrel, UK). Thermistors were calibrated across the physiological range against a certified 
reference thermometer (Dobros total immersion, Dobbie Instruments, Sydney, Australia).

\section{Design and analysis}

Several stages of data analysis were used. Firstly, the homogeneity of the sample was evaluated, with raw and heightadjusted morphological data compared across the body-mass classes (One-way Analysis of Variance). Tukey's HSD post hoc procedure was used to isolate significant differences. If inter-class differences existed, Analysis of Covariance was performed to identify significant interactions and to evaluate the need to include such variables as covariates when scaling. Alpha was set at 0.05 for all analyses. Data for metabolic scaling are reported as means with standard deviations (SD) and $95 \%$ confident intervals (CI).

The assumptions underlying linear and allometric scaling have both statistical and biological requirements. From a statistical perspective, distribution normality and linearity of both the raw (linear model) and log-linear or transformed data (allometric model), the variance of the residuals (homeoscedasticity) and first-order correlations (auto-correlations) were evaluated. Tests of normality were used with both the dependent variables (to evaluate the validity of the assumptions for linear regression) and the model residuals (to evaluate adherence to the assumptions of Analysis of Covariance). Those tests included: skewness and kurtosis $z$-scores, and the Shapiro-Wilk test (Shapiro and Wilk 1965). For the current sample size, $z$ scores within the range -3.29 to +3.29 corresponded with a normal distribution at the $5 \%$ probability level (Kim 2013). Scatter and residual plots were used to assess linearity and homeoscedasticity (respectively), and first-order correlations were investigated using the DurbinWatson test. The primary biological scaling criterion was the presence of an origin intercept, and that was evaluated using $t$-tests.

Two further analyses were performed before scaling, to minimise errors within the models. Firstly, Cook's distance test was used to evaluate apparently outlying observations that might disproportionately influence scaling. If the removal of those data significantly affected a scaling model (Analysis of Covariance), then those data were omitted. Secondly, bootstrapping was used to predict the most-likely coefficients for each of the regressions. Each regression was generated 1000 times using a re-sampling approach, from which the coefficient means and $95 \%$ confidence intervals could be derived and evaluated. Those confidence intervals were determined using Bias Corrected and Accelerated analyses, which accounts for imbalances either side of the mean, and provides a more likely confidence range.

Prior to scaling, the raw data were log transformed using a $\log _{10}$ base (log linear), enabling the non-linear model to be assessed using robust statistical comparisons that assumed linearity. The validity of that approach was determined using the coefficient of determination $\left(r^{2}\right)$ of the log-linear format. However, unless stated otherwise, allometric scaling was performed using untransformed data, as is the convention, and because errors can be introduced when applying an equation generated using transformed data to scale untransformed data, or to predict metabolic rate from body mass (Packard et al. 2011; Xiao et al. 2011).

Two regression models were used to evaluate the body-mass dependency of the metabolic data; linear regression (first-order polynomial regression; Eq. 1a) and non-linear (allometric) regression of the untransformed (raw) data (Eq. 2a). When the scaling exponent $(k)$ of Eq. 2a equals one, the relationship is linear (isometric); for all other exponents, the relationship is allometric. The possibility was explored (but not reported) that a more complex allometric model (the addition of an ordinate intercept into Eq. 2a: metabolic rate $=b *$ mass $^{k}+c$ ) might yield superior curve fitting. However, that approach yielded a significant, and biologically invalid, negative ordinate intercept $\left(-4.570 \mathrm{MJ} \mathrm{day}^{-1}\right)$. To permit a statistical evaluation of data used within Eq. 2a, non-linearity was removed using a loglinear transformation (Eq. 3a). Differences between the linear coefficients (slopes) within the linear and log-linear models were compared using Analysis of Covariance. The presence of a significant interaction effect indicated that the two models could not be scaled using the same coefficient (non-linear: exponent). Where appropriate, differences in the goodness of fit between the linear and non-linear models were compared using the root-mean-square error (RMSE), the Akaike Information Criterion (AIC), $r^{2}$ and $F$-tests (Motulsky and Ransnas 1987). The resulting human, allometric exponent was then compared (using $t$ tests) with exponents determined for other mammals, both within and well beyond the human bodymass range: dogs (mass ${ }^{0.885}$; Heusner 1991), pigs $<140 \mathrm{~kg}$ (mass $^{0.60}$; Noblet et al. 1994) and a very large inter-specific sample (mass ${ }^{0.67}$; White and Seymour 2005). The following general equations appear again within the results, bearing the same numerical codes, but different alphabetical qualifiers to signify changes in their use, and the resulting coefficients or exponents.

Linear regression : Metabolic rate $=a+b *$ mass $\left[\mathrm{MJ} \mathrm{day}^{-1}\right]$

where $a$, ordinate intercept [MJ day $\left.{ }^{-1}\right] ; b$, linear coefficient [slope; $\mathrm{MJ} \mathrm{kg}^{-1} \mathrm{day}^{-1}$ ].

Allometric regression : Metabolic rate $=b * \operatorname{mass}^{k} \quad\left[\mathrm{MJ}\right.$ day $\left.^{-1}\right]$

where $b$, linear coefficient [ $\mathrm{MJ} \mathrm{kg}^{-1} \mathrm{day}^{-1}$ ]; $k$, coefficient of allometry (scaling exponent) for body mass $[\mathrm{kg}]$. 
Log-linear : $\log _{10}($ metabolic rate $)$

$$
=\log _{10}(b)+k * \log _{10}(\text { mass })\left[\mathrm{MJ} \mathrm{day}^{-1}\right]
$$

where $b$, linear coefficient $\left[\mathrm{MJ} \mathrm{kg}^{-1} \mathrm{day}^{-1}\right] ; k$, scaling exponent for body mass $[\mathrm{kg}]$.

\section{Results}

\section{Homogeneity of the experimental sample}

Despite more than a two-fold range in body mass (56.0-117.1 kg), the experimental participants were not significantly different in either their height-to-mass proportionality or their subcutaneous adiposity (Table $1 ; P>0.05$ ), except when the two classification extremes were compared $(P<0.05$; extra-small versus extra-large). That is, both height-adjusted body mass and adiposity tended to overlap across those groups (Table 1), and that was interpreted to indicate that, to a very large extent, and within the limitations of those methods, the influence of variations in body composition on basal metabolism would be minimal. Moreover, there were no significant covariant influences on the scaling relationships $(P>0.05)$.

\section{Integrity of the experimental basal conditions}

Absolute, basal oxygen consumption data for five experimental participants (one from each mass class) are shown in Fig. 1, revealing within-subject stability and a body-mass

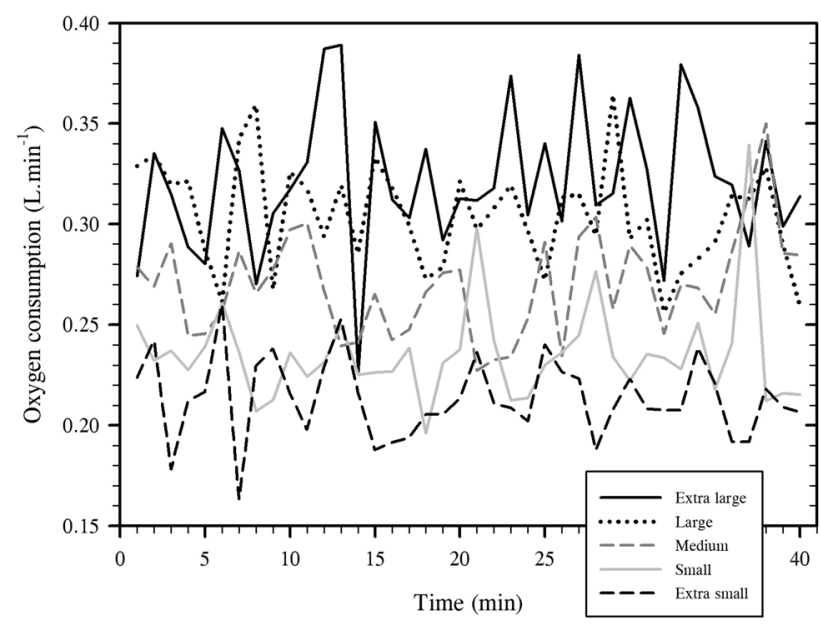

Fig. 1 Continuous, basal oxygen consumption data from five experimental participants taken from each of the five body-mass classifications. Data are 15-s averages collected over $60 \mathrm{~min}$ of supine rest under normothermic conditions (see "Methods"). The first 15 min of data were discarded (i.e., time zero starts at $16 \mathrm{~min}$ ), as well as the last $5 \mathrm{~min}$ bias. Across all subjects, the intra-individual coefficient of variance $(\mathrm{CV})$ for basal oxygen consumption was $12 \%$ (SD 0.04), for heart rate it was 7\% (SD 0.03) and for deep-body temperature $0.001 \%$ (SD 0.001). Since the oxygen consumption variance was $<20 \%$, it was interpreted that those data reflected stable and valid estimations of metabolic rate (McClave et al. 2003). Moreover, the mean respiratory exchange ratio was 0.87 , with a $\mathrm{CV}$ of $5 \%$ (SD 0.02), indicating normal breathing patterns and mixed-substrate oxidation consistent with the post-absorptive state. Those outcomes validated use of the Weir (1949) formula, with the basal metabolic rate of the experimental sample averaging $8.065 \mathrm{MJ} \mathrm{day}^{-1}$ (SD 0.971; mean body mass $81.6 \mathrm{~kg}$ [SD 14.7]). Furthermore, inter-class differences in the $\mathrm{CV}$ were not observed for either oxygen consumption (extra small, $11 \%$ [SD 0.02]; small, $13 \%$ [SD 0.05]; medium, $12 \%$ [SD 0.04]; large, 13\% [SD 0.05]; extra large, 13\% [SD 0.04]) or deep-body temperatures (all $<0.5 \%$ ), demonstrating consistency of the impact of that thermoneutral environment, regardless of the thermal inertia of those individuals.

\section{The assumptions of metabolic scaling}

The ability to normalise metabolic data from the experimental dataset was assessed using two linear scaling methods: ratiometric (arithmetic) analysis and linear regression (Eq. 1a). The latter model was used to normalise data using both intercept and slope adjustments (after Albrecht et al. 1993). Linear regression relies on four statistical assumptions, which could also be evaluated for allometric scaling using log-linear (transformed) data.

Distributions of basal metabolic rate and body mass were assessed for normality and linearity using both raw (untransformed; linear regression; Fig. 2a) and log-linear data formats (Fig. 2c; logarithmic scale). Skewness and kurtosis $z$ scores were well inside the acceptable range $(P>0.05$; Table 2), and the Shapiro-Wilk test was not significant $(P>0.05$; Table 2$)$, confirming those data were normally distributed. Residual plots were used to assess homeoscedasticity, with both the raw and log-linear data satisfying that requirement (Fig. 2b and d, respectively). Finally, first-order correlations were not observed within the residuals (DurbinWatson test $P>0.05$; Table 2). Therefore, despite metabolic rate and body mass being inherently related, they were not inter-dependent. Consequently, the application of both the linear and allometric scaling methods to those experimental data was statistically justified.

Perhaps the most prevalent form of physiological normalisation involves ratiometric analysis (division by body mass; the ratio standard). That method frequently converts a mass-dependent bias (positive; Fig. 2a; $r=0.82$ ) into a systematic negative bias (Fig. 3a; $r=-0.75$ ), invalidating its use for the experimental participants (Albrecht et al. 1993). 

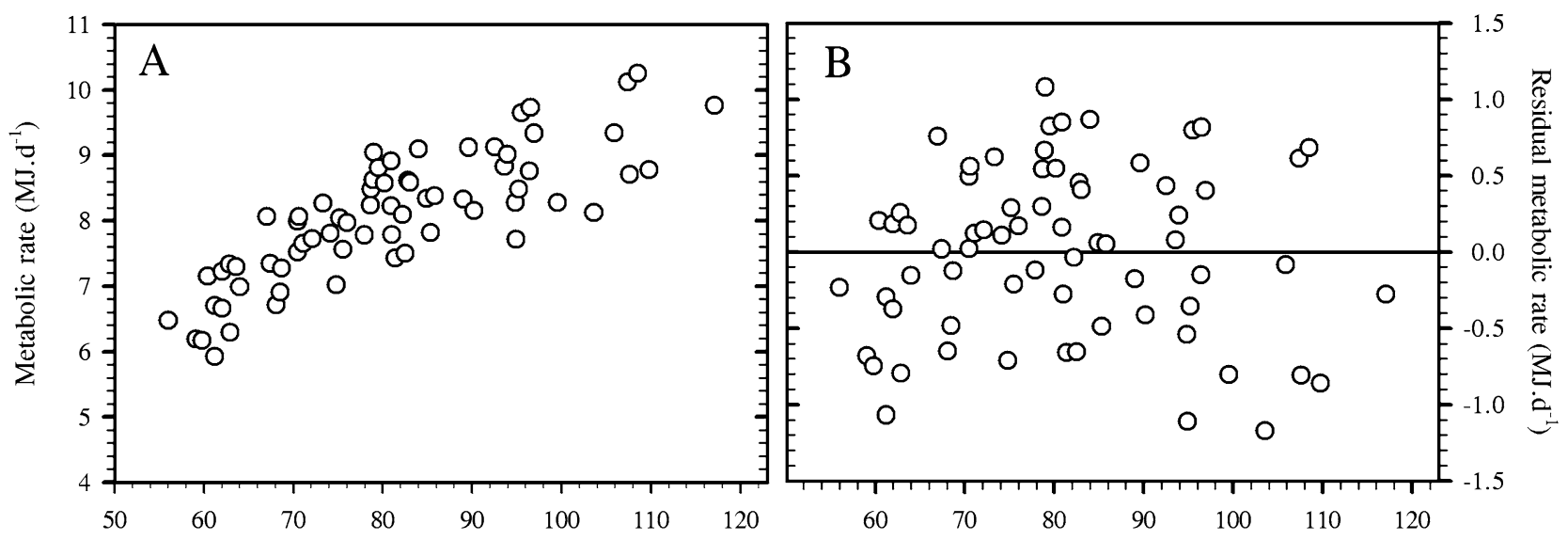

Body mass $(\mathrm{kg})$
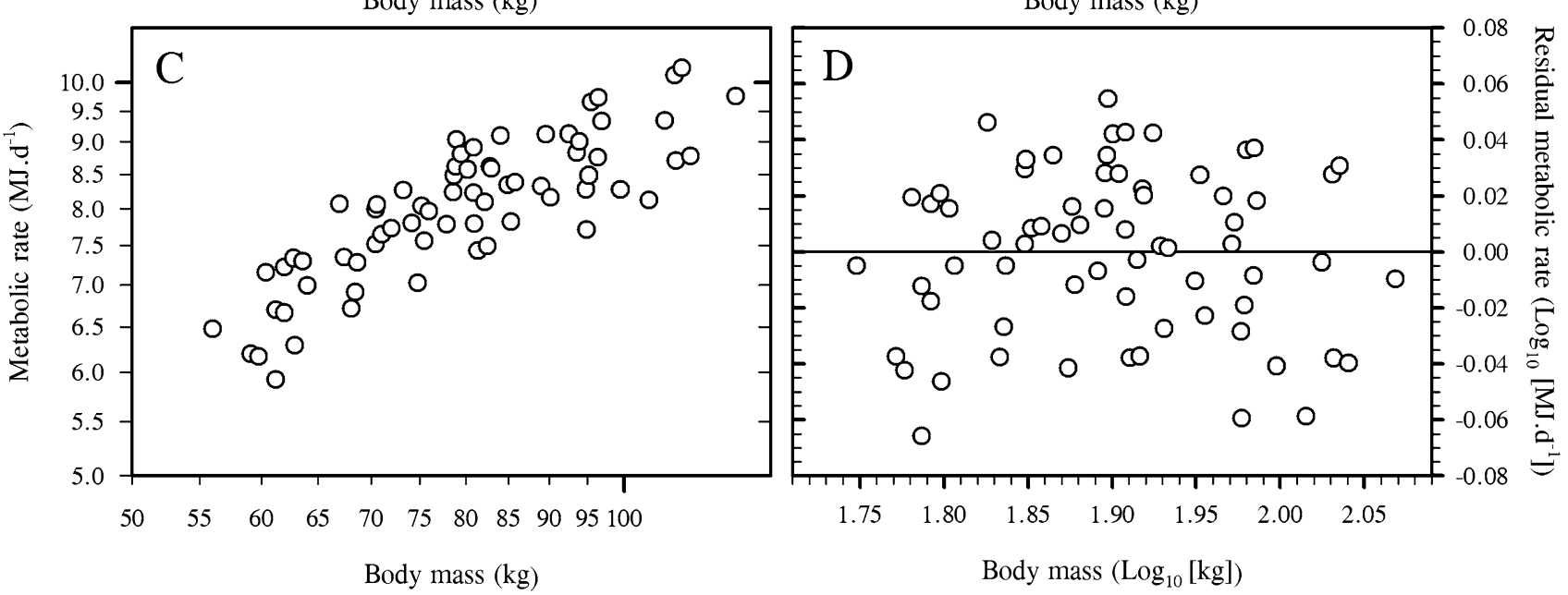

Fig. 2 Scatter plots used to assess the linearity of the basal metabolic rates of the experimental participants $(N=68)$, with absolute values presented on both linear (a) and logarithmic scales (c). Also presented are the corresponding residual plots used to assess the home-

oscedasticity of linear and non-linear scaling (b and $\mathbf{d}[\log$ transformed data], respectively). Those residuals are differences between the measured metabolic rates and values derived using predictions applied to data from $\mathbf{a}$ and $\mathbf{c}$ (respectively)

Table 2 Tests of normality and first-order correlations (auto-correlation) between the dependent (basal metabolic rate) and independent variables (body mass) for the experimental dataset $(N=68)$

Dependent and independent variables

\begin{tabular}{|c|c|c|c|}
\hline \multicolumn{2}{|c|}{ Basal metabolic rate } & \multicolumn{2}{|l|}{ Body mass } \\
\hline $\operatorname{Raw}\left(\mathrm{MJ} \mathrm{day}^{-1}\right)$ & $\begin{array}{l}\text { Transformed }\left(\log _{10}\right. \\
\left.\left[\mathrm{MJ} \mathrm{day}^{-1}\right]\right)\end{array}$ & Raw (kg) & $\begin{array}{l}\text { Transformed } \\
\left(\log _{10}[\mathrm{~kg}]\right)\end{array}$ \\
\hline
\end{tabular}

\begin{tabular}{lcccrrr}
\hline Skewness statistic & -0.06 & -0.37 & 0.40 & 0.07 & -0.19 & -0.33 \\
$z$ score & -0.21 & -1.27 & 1.36 & 0.24 & -0.67 & -1.12 \\
Kurtosis statistic & 0.42 & -0.16 & -0.56 & -0.79 & -0.77 & -0.73 \\
$z$ score & 0.47 & -0.16 & -0.97 & -1.60 & -0.35 & -1.27 \\
Shapiro-Wilk statistic & 0.99 & 0.98 & 0.97 & 0.98 & 0.98 & 0.97 \\
Durbin-Watson statistic & - & - & - & - & 1.63 & 1.68 \\
\hline
\end{tabular}

Data were analysed in both raw and $\log _{10}$-transformed formats. None of those tests revealed significant differences at the $5 \%$ probability level A perfect, normal distribution would have a Shapiro-Wilk test statistic of 1.00 (Shapiro and Wilk 1965), while the complete absence of autocorrelation between the dependent and independent variables would have a Durbin-Watson test statistic of 2.00 (Durbin and Watson 1950, 1951) 

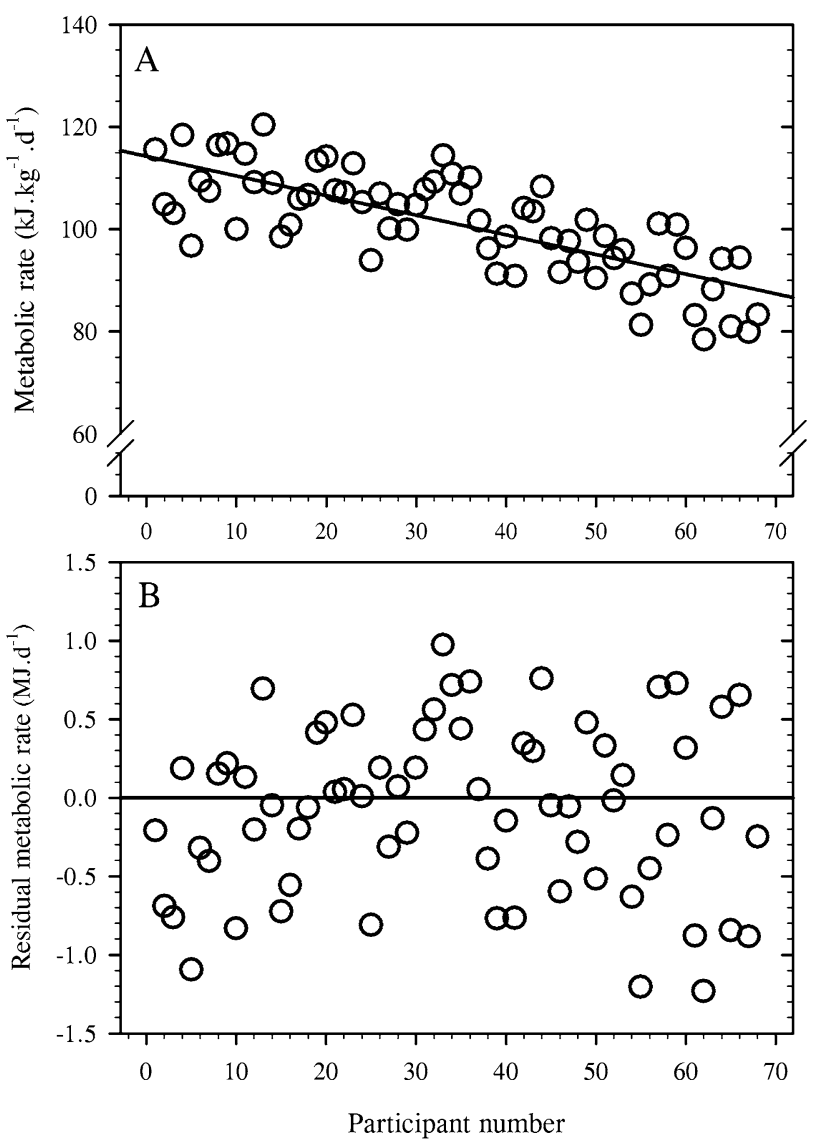

Fig. 3 Relationships between basal metabolic rate and body mass within the experimental participants, arranged from lightest (leftmost) to heaviest across the abscissae $(N=68)$. In a, the absolute metabolic rate for each individual was converted to its mass-specific equivalent using ratiometric scaling $(r=-0.75 ; P<0.05)$. b Contains residuals derived from differences between the measured and predicted absolute metabolic rates, with those predictions derived using allometric scaling (Eq. 2B; $r=0.02 ; P>0.05$ ). These plots provide a visual evaluation of the extent to which that procedure removed the mass bias from the basal metabolic data

On the other hand, that method satisfied the biological assumption (origin intercept), but it provided an inferior fit $(\mathrm{RMSE}=0.849, \mathrm{AIC}=174.72)$, with significant metabolic over-predictions beyond $95 \mathrm{~kg}$, and under-predictions for masses $<55 \mathrm{~kg}$ (Fig. 4). To highlight that problem, ratiometric mass-specific metabolic rates were compared between two sub-groups; the ten smallest (60.7 kg [SD 2.1]) and the ten largest individuals (105.3 $\mathrm{kg}$ [SD 6.4]). That comparison revealed significantly different mass-specific metabolic rates $\left(87.7 \mathrm{~kJ} \mathrm{~kg}^{-1}\right.$ [SD 2.5] and $108.9 \mathrm{~kJ} \mathrm{~kg}^{-1}$ [SD 2.4], respectively; $P<0.05$ ), verifying that, even across the adult mass range, basal metabolic rates were body-mass dependent and non-linear. Accordingly, ratiometric scaling of the experimental data was unacceptable, and not considered further.

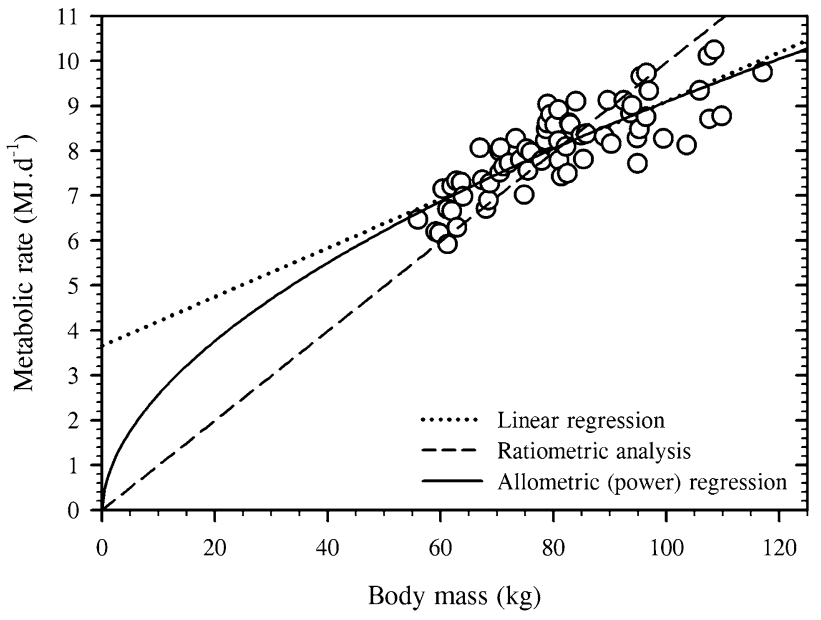

Fig. 4 Modelling the relationship between absolute, basal metabolic rate and body mass within the experimental subjects $(N=68)$ using three scaling methods: linear regression (least-squares, firstorder polynomials), ratiometric analysis and allometric regression (Eq. 2b). The absence of an origin intercept (3.659 $\mathrm{MJ} \mathrm{day}^{-1}$ ) for the linear model is thermodynamically impossible, violating the biological assumption underpinning all scaling models, even though it provided a strong correlation over the mass range investigated $\left(r^{2}=0.68\right.$; $P<0.05$ ). For body masses $<55 \mathrm{~kg}$, that model would yield metabolic over-predictions of progressively increasing magnitude. Ratiometric modelling would produce over-predictions for body masses $>95 \mathrm{~kg}$, and under-predictions for masses $<55 \mathrm{~kg}$

A consequence of applying linear scaling to non-linear data is that the ordinate intercept infrequently passes through the origin. Non-zero metabolic intercepts are biologically impossible within healthy individuals. When least-squares, linear regression analysis was applied to the experimental data, that biological impossibility occurred (Fig. 4), resulting in an ordinate intercept of $3.659 \mathrm{MJ} \mathrm{day}^{-1}$ (confidence interval: 2.879-4.482), and thereby invalidating linear modelling for data beyond the experimental body-mass range. Nonetheless, within the body-mass range investigated, that method provided both a visually appropriate and statistically strong correlation between the variables of interest (Fig. $4 ; r^{2}=0.68$ [coefficient of determination]; $P<0.05$, RMSE $=0.549$; $\mathrm{AIC}=117.45$; Eq. 1b). Moreover, it removed the mass bias from the measured metabolic data [see: Albrecht et al. (1993) Eq. 3], as demonstrated by the correlation coefficient for the residuals (Fig. 2b, $r=0.03 ; P>0.05$ ). However, the capacity to predict (and normalise) metabolic rates beyond that bodymass range remained limited and produced over-predictions of progressively increasing magnitude as mass decreased (Fig. 4). Accordingly, linear regression was also considered to be unacceptable.

Metabolic rate $=3.659\{2.879-4.482\}$

$$
+0.054\{0.044-0.064\} * \text { mass }
$$


where metabolic rate units are $\mathrm{MJ} \mathrm{day}^{-1}$ and body mass is in $\mathrm{kg}, 95 \%$ confidence intervals are shown within the braces.

\section{Allometric scaling of basal metabolic rate: experimental data}

Having satisfied the statistical pre-requisites using log-linear data, two non-linear methods were applied to the basal metabolic data of the experimental subjects; simple allometric (power) scaling of the untransformed (raw) data and linear scaling of the log-linear data. The latter was performed to facilitate statistical analyses. The former yields a curve that passes through the origin, with the resulting prediction equation (Eq. $2 \mathrm{~b}$; a power standard) providing a biologically valid, powerful and statistically significant description of the relationship between basal metabolic rate and body mass $(P<0.05$; RMSE $=0.538$, AIC $=114.76)$. Not surprisingly, when presented graphically (Fig. 3b), those data had the lowest correlation with body mass $(r=0.02 ; P>0.05)$. For inter-individual comparisons of previously measured metabolic rates, Eq. $2 \mathrm{~b}$ can simply be rearranged to adjust absolute values $(Y)$ to their mass-specific equivalents $\left(Y_{\text {adjusted }}=Y /\right.$ mass $\left.^{k}\right)$.

Metabolic rate $=0.739\{0.489-1.061\} *$ mass $^{0.547\{0.461-0.639\}}$

where metabolic rate units are $\mathrm{MJ}$ day $^{-1}$ and body mass is in $\mathrm{kg}, 95 \%$ confidence intervals are shown within the braces.

Plotting those data on logarithmic scales (Fig. 2c), and using linear modelling (Eq. 3b), provided a valid evaluation of the goodness of fit of the allometric model $\left(r^{2}=0.70\right)$. It also facilitated subsequent statistical comparisons between the experimental allometric model and similar models generated using the historical datasets. From this evidence, it was apparent that both linear regression and the allometric model provided similar visual and statistical fits for the experimental data $(P>0.05)$

$$
\begin{aligned}
\log _{10}(\text { metabolic rate })= & -0.181\{-0.370 \text { to }-0.002\} \\
& \left.+0.570\{0.474-0.668\} * \log _{10} \text { (mass }\right)
\end{aligned}
$$

where metabolic rate units are $\mathrm{MJ}$ day $^{-1}$ and body mass is in $\mathrm{kg}, 95 \%$ confidence intervals are shown within the braces.

\section{Allometric scaling of resting metabolic rate: historical database}

Ratiometric, linear regression and allometric regression analyses were now applied to the historical database (males), using the 101 body-mass classes contained therein. Firstly, those methods were used to predict the absolute metabolic rate for each body-mass class. The ratiometric prediction was obtained from the product of body mass and the bootstrapped linear coefficient (slope) obtained from those historical data. For the linear modelling, absolute metabolic rate was predicted using the bootstrapped intercept and slope computed using least-squares, best-fit linear regression (Eq. 1a). Similarly, an allometric prediction of absolute metabolic rate was derived using the bootstrapped allometric coefficient and scaling exponent calculated from those data (Eq. 2a). Secondly, to evaluate the validity of each metabolic prediction, those values were subtracted from the measured metabolic rates, with the resulting residuals presented in Fig. 5. As expected, the ratiometric predictions displayed a body-mass bias for masses $>20-30 \mathrm{~kg}$ (Fig. 5a). The linear metabolic predictions were much less mass dependent (Fig. 5b), but considerably more variable than the allometric predictions (Fig. 5c). Accordingly, those linear methods were not further considered. Nevertheless, for body masses $<10 \mathrm{~kg}$, the allometric predictions were unreliable and revealed a systematic bias with predictive errors between 150 and $300 \%$. The cause of that bias remains uncertain. It could reflect reality, but it may be associated with smaller sample sizes within the body-mass bins $<10 \mathrm{~kg}$ (average size: 15) or with reduced experimental control within the infants and children. For instance, the coefficient of variation $(\mathrm{CV})$ of the resting metabolic rate was $21.2 \%$ across those individuals. For individuals between 50 and $70 \mathrm{~kg}$, the average sample size was 141 and the $C V$ was $9.8 \%$. Beyond $85 \mathrm{~kg}$, bias was not evident, but there was much greater inter-individual noise. Now the sample sizes averaged less than three individuals, and the $\mathrm{CV}$ was $7.0 \%$.

The emphasis now turns wholly to the allometric scaling (Eq. 2a) of the historical dataset, which is presented in Fig. 6 in two forms. Firstly, the complete dataset was independently scaled (Fig. 6a: males), yielding a powerful prediction (Eq. 2c; $P<0.05$ ). Secondly, to evaluate how well the basal metabolic rates of the 68 experimental participants compared with those historical data, the former were overlayed (Fig. 6b), and included within another allometric analysis (179 data points). The experimental data were individual data points, rather than group means, and were therefore more variable. Nevertheless, they conformed with those data, producing a powerful overall prediction (Eq. 2d; $P<0.05$ ), with Eqs. $2 \mathrm{c}$ and $2 \mathrm{~d}$ not differing significantly $(P>0.05)$. That is, although those equations were numerically different, they were statistically equivalent, contained the same information and would yield small, non-significant differences in predicted metabolic rate. This was important because a single exponent $(0.55)$ could now be used to describe the intra-specific relationship between basal and resting metabolic rates and body mass within healthy humans. Nonetheless, when compared with data obtained from other mammals, that exponent was statistically smaller than the exponents derived from the inter-specific scaling of mammalian metabolic rates (body mass ${ }^{0.67}[P<0.05]$; 

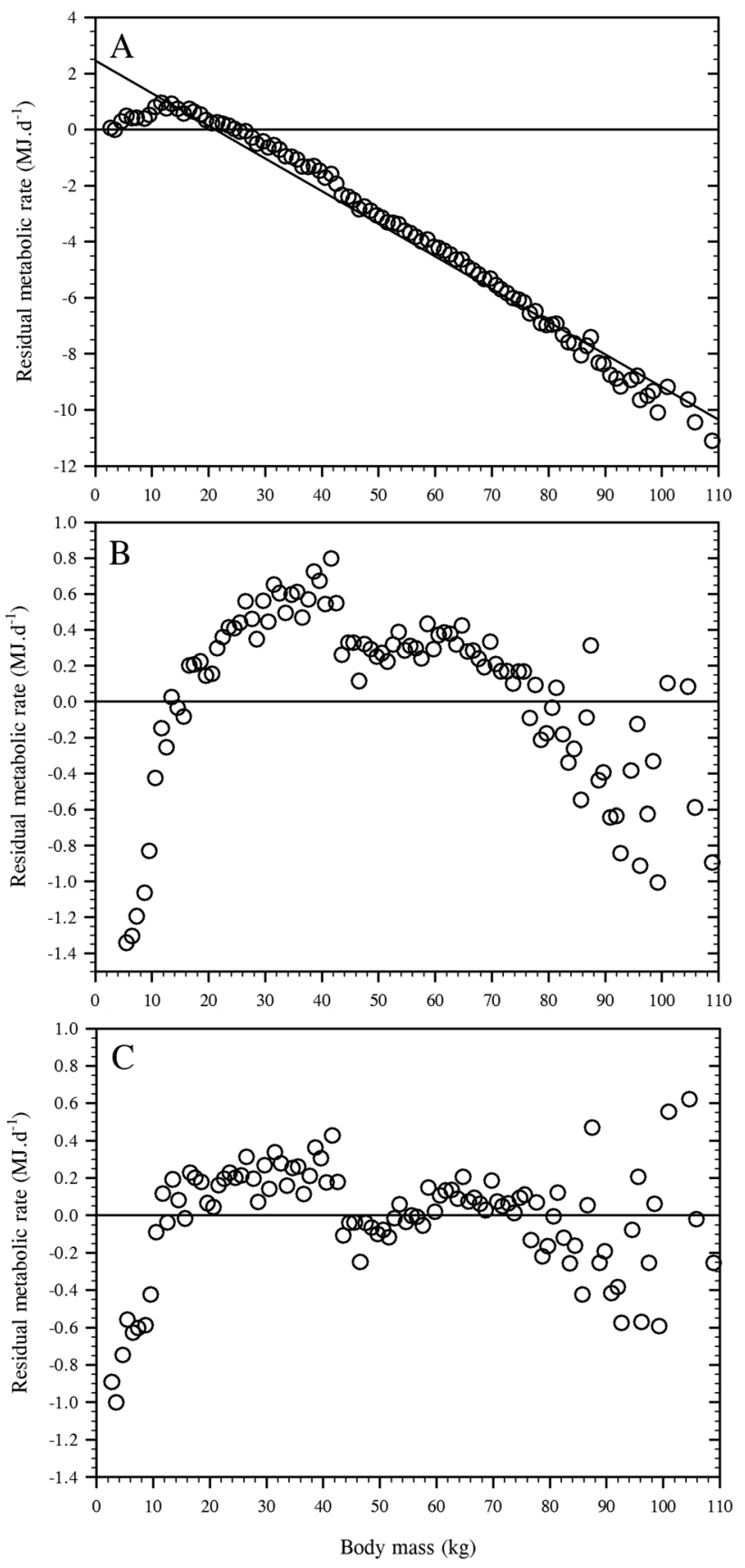

Fig. 5 Relationships between the measured and predicted absolute, resting metabolic rate and body mass for males $(N=4811)$ from the historical database (Schofield 1985). Data are residuals (measured minus predicted) presented in $1-\mathrm{kg}$, body-mass classes (101 means). Three scaling methods are illustrated: ratiometric $(\mathbf{a} ; r=-0.77$; $P<0.05)$, linear $(\mathbf{b} ; r=0.07 ; P>0.05)$ and allometric scaling $(\mathbf{c}$; Eq. $2 \mathrm{~B} ; r=0.03 ; P>0.05)$

White and Seymour 2005), and also from the intra-specific scaling of canine metabolism (mass ${ }^{0.885}[P<0.05]$; Heusner 1991). However, it did not differ significantly from another intra-specific (porcine) scaling model (mass ${ }^{0.60}[P>0.05]$;
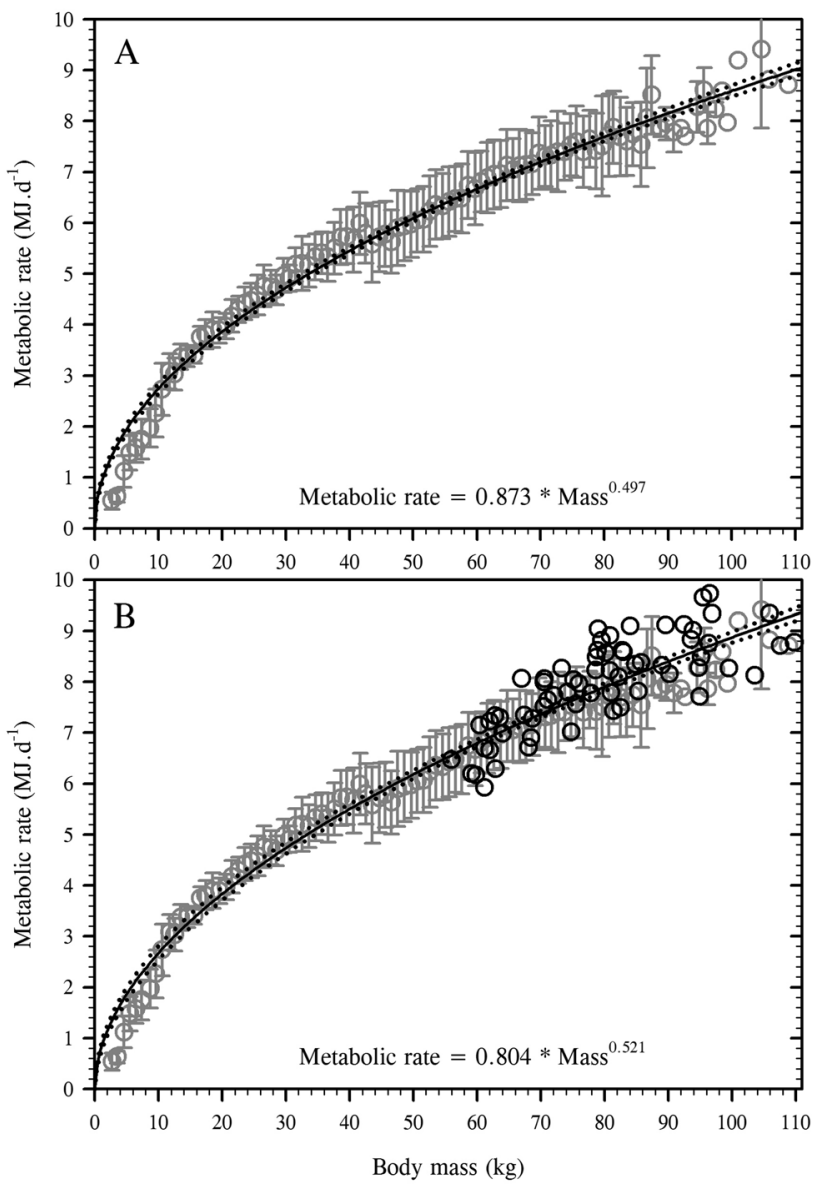

Fig. 6 Allometrically scaled resting, absolute metabolic rates (males; $N=4811$ ) from the historical database (Schofield 1985; a; $P<0.05$ ). Those data were pooled into $1-\mathrm{kg}$, body-mass classes (grey symbols), and are presented as 101 means with standard deviations (where available). The solid (black) line is the allometric curve for those data (Eq. 2C), with 95\% confidence intervals (dotted curves). In addition, the mean, basal metabolic rates of each experimental subject were overlayed onto the historical data (b; open, black circles; $N=68)$, and both datasets were collectively scaled (Eq. 2D; $P<0.05 ; 179$ means representing 4879 individuals)

Noblet et al. 1994). These observations are consistent with the evidence that a universal (allometric) scaling exponent does not exist (Heusner 1982a; White and Seymour 2003; Sieg et al. 2009).

Metabolic rate $=0.873\{0.790-0.956\} * \operatorname{mass}^{0.497\{0.474-0.520\}}$

Metabolic rate $=0.804\{0.730-0.878\} *$ mass $^{0.521\{0.499-0.544\}}$

where metabolic rate units are $\mathrm{MJ} \mathrm{day}^{-1}$ and body mass is in $\mathrm{kg}, 95 \%$ confidence intervals are shown within the braces.

To evaluate the robustness of Eq. 2c, two samples were randomly extracted from the historical (male) database (random-number generator), yielding unique sub-sets containing 
$50(N=2099)$ and $51(N=2712)$ of the original $101(1-\mathrm{kg})$ mass classifications. Each sub-set was separately scaled (Fig. 7), generating two equally powerful predictions (Eq. 2e $[P<0.05$; Fig. 7a] and Eq. 2f $[P<0.05 ;$ Fig. 7b]), which did not differ significantly from Eq. $2 c(P>0.05)$. Consequently, Eq. $2 \mathrm{c}$ was deemed to be both a valid and robust predictive model suitable for use within the wider population of males within the body-mass range of $10-85 \mathrm{~kg}$.

Metabolic rate $=0.906\{0.795-1.024\} *$ mass $^{0.487}\{0.458-0.518\}$

Metabolic rate $=0.807\{0.713-0.960\} *$ mass $^{0.506\{0.473-0.544\}}$

where metabolic rate units are $\mathrm{MJ} \mathrm{day}^{-1}$ and body mass is in $\mathrm{kg}, 95 \%$ confidence intervals are shown within the braces.

As the historical dataset also contained resting metabolic data for an independent sample of females $(N=2364$; Schofield, 1985), the opportunity was taken to explore gender dependency within the relationship describing body mass and metabolic rate. The separately generated male (Fig. 6A; Eq. 2c) and female (Eq. $2 \mathrm{~g} ; P<0.05)$ scaling models, both of which were derived using the complete datasets, did not differ significantly $(P>0.05$; Fig. $8 b)$, supporting the possibility of gender independence across those body-mass ranges. Nevertheless, above $40 \mathrm{~kg}$, data divergence was apparent. That possibility was explored by applying allometric scaling only to data from the body-mass classifications from $40 \mathrm{~kg}$ through to $96 \mathrm{~kg}$ within each dataset (Fig. 8c; 54 classes from each gender; $N=3871$ [males] and $N=1443$ [females]). The lowest average ages for those mass classes were 17.6 and 18.4 years (respectively), closely approximating that of the experimental sample. The resulting equations now differed significantly $(P<0.05$; females Eq. $2 \mathrm{~h}$ and males Eq. 2i), confirming a gender-dependent difference within the resting metabolic rate of adults with the same body mass.

Metabolic rate (all females)

$$
=0.961\{0.837-1.084\} * \operatorname{mass}^{0.435\{0.405-0.468\}}
$$

Metabolic rate (female $40-96 \mathrm{~kg}$ )

$$
=1.094\{0.922-1.286\} * \operatorname{mass}^{0.402\{0.362-0.444\}}
$$

Metabolic rate (male $40-96 \mathrm{~kg}$ )

$$
=1.012\{0.873-1.168\} * \operatorname{mass}^{0.461\{0.426-0.497\}}
$$

where metabolic rate units are $\mathrm{MJ}$ day $^{-1}$ and body mass is in $\mathrm{kg}, 95 \%$ confidence intervals are shown within the braces.
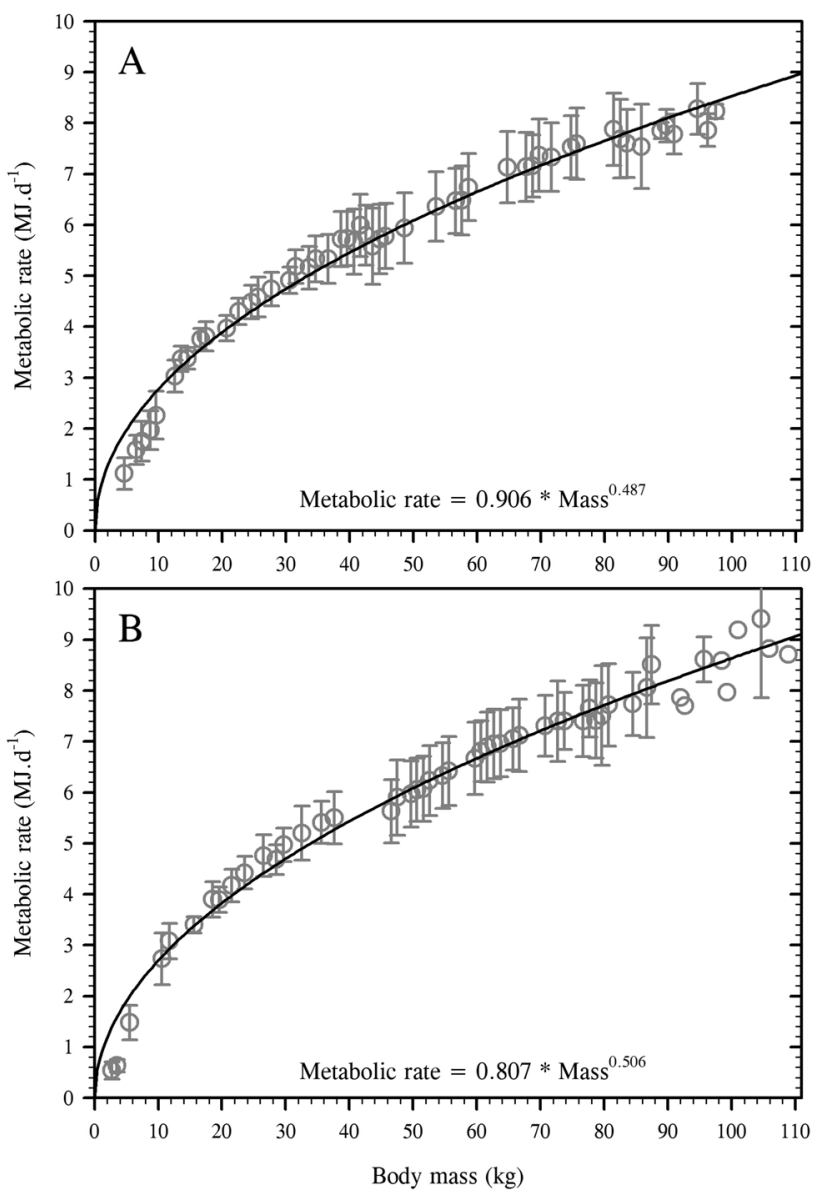

Fig. 7 Allometric scaling of the resting, absolute metabolic rates (males) for two sample sub-sets, randomly extracted from the historical database (Schofield 1985), and analysed separately. a Contains 50 body-mass classes $(N=2099$; Eq. 2E) whilst b shows another (unique) 51 mass classes $(N=2712$; Eq. $2 \mathrm{~F}])$. Data are means with standard deviations (where available), with the black curves showing the resulting allometric relationships (both $P<0.05$ )

\section{Discussion}

This descriptive experiment has revealed several novel and important outcomes. Firstly, it has established, perhaps unequivocally, that basal and resting metabolic rates scale allometrically with body mass in healthy humans. Secondly, when combined with the extensive (resting) metabolic data for healthy males and females contained within an historical database (Schofield 1985), and extending down to masses $<3 \mathrm{~kg}$, that scaling method effectively removed the mass bias from those data for masses between 10 and $85 \mathrm{~kg}$. Thirdly, when independent analyses were applied to two unique and randomly chosen (male) sub-groups from that database, statistically equivalent allometric outcomes were produced, establishing the robustness of that allometric 

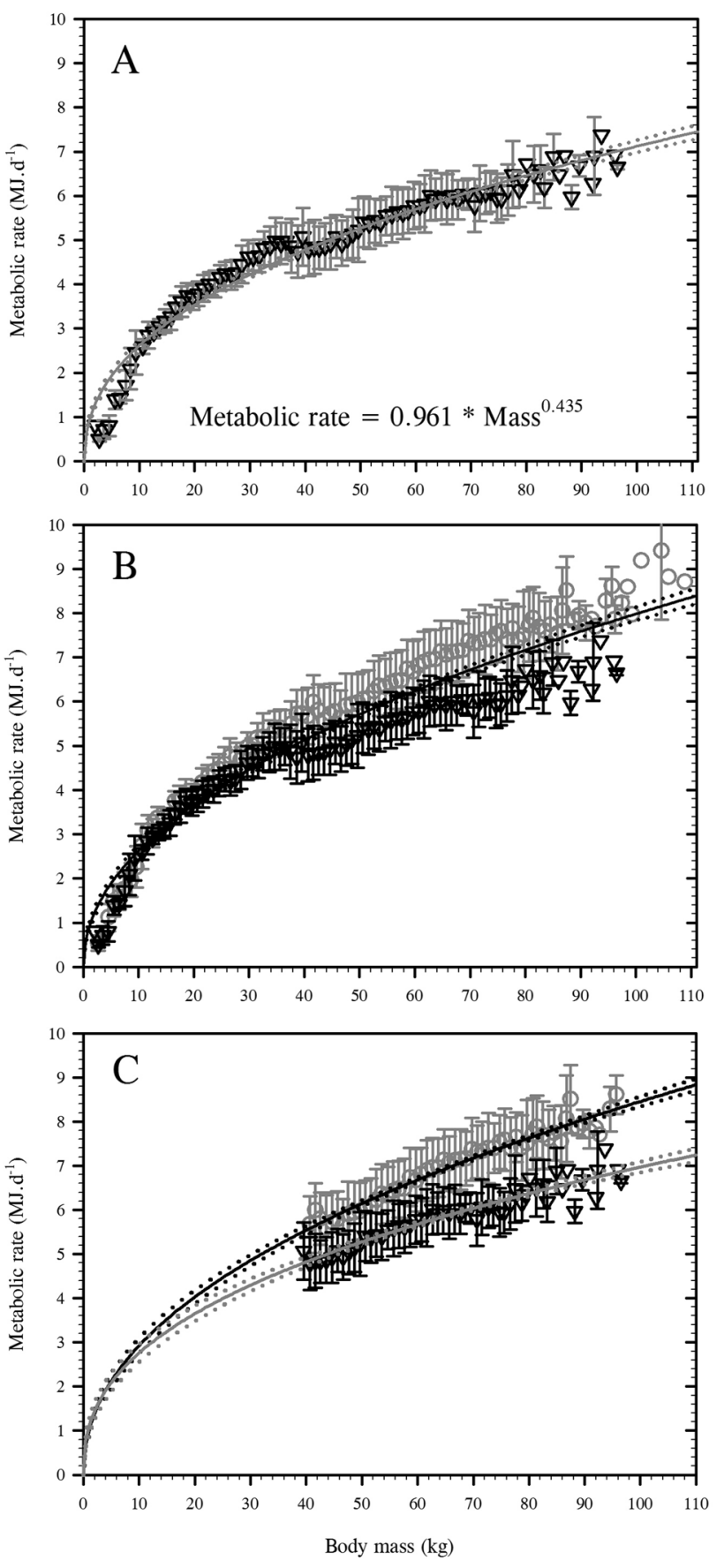

Fig. 8 Allometric scaling of the resting, absolute metabolic rates of females $(N=2364)$ from the historical database $(\mathbf{a}$; Eq. $2 \mathrm{~g} ; P<0.05$; Schofield 1985). Data were pooled into 94 body-mass classes, each of which represented a $1-\mathrm{kg}$ mass increase (black triangles), and are presented as means with standard deviations (where available). The grey curve shows the allometric relationship, with $95 \%$ confidence intervals (dotted curves). b Contains the corresponding male data from Fig. 6a (grey circles) with the female data from a (black triangles). Collectively, those 196 means represent 7175 individuals, which were combined and scaled to produce a single allometric model (black curve with $95 \%$ confidence intervals [dotted curves]). In c, gender-specific data were extracted for masses between 40 and $96 \mathrm{~kg}$, and separately modelled. The resulting allometric curves are shown (Eq. $2 \mathrm{~h}$ [females: triangles with grey curve] and Eq. 2i [males: circles with black curve]), along with $95 \%$ confidence intervals (dotted curves) model. Those outcomes justified acceptance of the working hypothesis; both the basal and resting metabolic rates of healthy humans scale allometrically with body mass. Fourthly, the resulting mass exponents were consistently between 0.50 and 0.55 , whereas inter-specific exponents (typically 0.67; White and Seymour 2005) are 20-30\% larger $(P<0.05)$. Finally, when males and females from those historical data (Schofield 1985) were compared over the adult age range ( $>18$ years), as well as across an equivalent bodymass range (40-96 kg), statistically significant differences in the mass exponents resulted (mass ${ }^{0.46}$ [males] and mass ${ }^{0.40}$ [females]), implying a gender-dependent difference in the mass-specific, resting metabolic rate of adults.

\section{Linear or non-linear: that is the question}

Two opposing scaling methods have been used to describe human metabolic data: linear (Harris and Benedict 1919; Schofield 1985; Cole and Henry 2005) and allometric scaling (Boothby and Sandiford 1922; Rogers et al 1995; Markovic et al. 2007). In many cases, linear models were more expedient, and, as demonstrated above, can be used to adequately model data over a limited mass range. Beyond that range, however, such models are predictably unreliable when applied to data that is inherently non-linear (Fig. 4), and "the cost of 'simplicity' would need to be estimated" (Schofield 1985; P12). Two of those costs are immediately apparent; an inability to attain a truly mass-independent relationship and the lack of precision for metabolic predictions outside the experimental body-mass range. Let us firstly consider mass independence. To determine whether linear or allometric scaling might be superior, it was necessary to evaluate both the statistical justification and the biological validity of those methods.

It is insufficient to address only mathematical precision, since an inability to reflect biological reality invalidates any model, regardless of its closeness of fit; best fit does not necessarily equate with the correct solution (Sholl 1948; Motulsky and Ransnas 1987; Nevill et al. 1992). This is exemplified in Fig. 4, where best-fit lines for the linear and allometric regression solutions were effectively superimposed over the mass range of the experimental subjects, whilst the ratiometric relationship was appreciably different. Consequently, when the ratiometric, mass-specific relationship was illustrated for individuals of increasing body mass (Fig. 3a), it was not horizontal, the mass bias was retained and the scaling was inadequate (Albrecht et al. 1993). Such non-horizontal lines demonstrate that the relationship was in fact, non-linear (Packard and Boardman 1988). Furthermore, both linear solutions yielded increasingly greater errors when predicting absolute metabolic rates for masses progressively further away from the mass range of the participants (Fig. 4). 
The pivotal assumption of linear scaling is that both the independent and dependent variables change isometrically, as occurs within geometrically similar objects, although such relationships are infrequently found in biology (Packard and Boardman 1988). Indeed, metabolic isometry exists only when a straight line that adequately describes the relationship between metabolism and body mass, also passes through the origin (Packard and Boardman 1988). Thus, the primary biological criterion for scaling is the presence of an origin intercept, since non-zero intercepts violate the First Law of Thermodynamics, and are biologically impossible (Krogh 1916; Kleiber 1950). That criterion excluded linear regression (Fig. 4). Ratiometric scaling satisfied that requirement (Fig. 4), but retained a significant mass-specific bias (Fig. 3a), with that limitation representing the second cost of simplicity; an inability to predict metabolic rates beyond the range of the experimental data.

"[T] he true test of any method for the reduction of the metabolism of individuals of different size and shapes to comparable terms is its capacity for predicting unknown metabolism" (Harris and Benedict 1919; P182). Indeed, for mathematical generalisations to be valid and broadly applicable, their robustness must be established for values of the independent variable (body mass) beyond the experimental range (Kleiber 1961). Consider the complications of administering a life-saving, metabolism-dependent drug to a $200-\mathrm{kg}$ individual. From Fig. 3a, it is apparent that an overestimation of the therapeutic concentration would result if ratiometric analysis had been used, so that method was not further considered, leaving the linear and allometric scaling options.

The former often represent more complex solutions. Indeed, such linear models are available in the literature, and use unique combinations of body mass, height, age, fatfree body mass, gender and even menopausal status (e.g. Harris and Benedict 1919; Owen et al. 1987; Fedrix et al. 1990). Notwithstanding the apparent preferential use of such models, they have long been criticised as physiologically meaningless (Krogh 1916; Sholl 1948; Kleiber 1961), even though they can be quite precise when applied within the body-mass ranges over which data were collected. Such criticisms are not directed at the mathematical precision, but the physiological relevance of each coefficient, and its justification; “... it is unlikely that such a polynomial will serve any useful function as a description of the mechanism underlying our experimental results since we shall have to find a biological interpretation inductively for each parameter ..." (Sholl 1948, P244). For the current experiment, a much less complex linear equation was derived (Eq. 1b), which was biologically valid and adequately removed the mass bias.

However, since we know that mammalian basal metabolism scales allometrically with body mass (White and
Seymour 2003), then why would humans not follow the same general pattern? Figures 6,7 and 8 show that the resting (historical) metabolic rates were not linearly related to body mass. Therefore, linear scaling of those data must result in either overtly, or potentially, erroneous predictions, leading to spurious interpretations. Accordingly, it is recommended that allometric scaling be adopted, and separate male (Eqs. 2c and 2i) and female (Eqs. 2g and 2h) predictive equations have been derived. It is intriguing to note that, when Kleiber (1932) modelled the entire metabolic dataset of Harris and Benedict (1919), he also reported an allometric relationship, but with an exponent (mass ${ }^{0.6}$ ) that was distinctly different from his inter-specific value derived for mammals (mass ${ }^{0.74}$ ), and closer to the exponents determined within the current analyses (mass ${ }^{0.50-0.55}$ ).

Whilst the experimental data are both unique and important, the power of this experiment comes through the combination of those data with the historical data. On its own, allometric scaling of the experimental data provided a strong predictive tool (Eq. $2 \mathrm{~b}$; mass ${ }^{0.55}$ ), although it might be difficult to convince some of the validity of that prediction for masses lower than $56 \mathrm{~kg}$ (Fig. 4). But when those data were analysed with the historical male data, the resulting allometric equation (Eq. 2d; Fig. 6b) did not differ significantly from either Eq. 2b or 2c (Fig. 6a), supporting the interpretation that those equations provide valid, mass-independent models of human basal and resting metabolic rates.

Such information is essential to those who model human metabolism, as it adds another layer of precision to those calculations, as well as increasing the mass range over which predictions can reliably be performed. Furthermore, those who prescribe metabolic rate-dependent pharmaceutical preparations will now have a more biologically valid means through which those doses can be administered. Dieticians, who typically base dietary recommendations on surface-area or mass-ratio standards, will have another, perhaps superior, means through which to make recommendations. Other applications exist within the exercise domain, within which mass-dependent physiological responses are frequently compared during steady-state and maximal exercise. Finally, there exist workplace applications. For example, such knowledge allows one to determine the appropriate load distributions among workers of varying body mass, such that all workers might be exposed to metabolically equivalent workplace demands (Bowes et al. 2017).

It is prudent, however, to offer cautionary comments concerning the historical data (Schofield 1985). Indeed, a potentially significant limitation associated with using those data is that no effort was made to ensure data were drawn from individuals of the same developmental state, although that could not reasonably have been expected. The inclusion of infants is both fortuitous, since it extended the mass ranges down to $2.7 \mathrm{~kg}$ (males) and $2.0 \mathrm{~kg}$ (females), and potentially 
misleading, since age-dependent variations exist for the separate and combined masses of the most metabolically active organs (Holliday et al. 1967; Holliday 1971; Müller et al. 2011). For example, exponents for the mass-dependent metabolic rate of some of those organs vary considerably in adults: mass ${ }^{0.26}$ (brain) to mass ${ }^{0.70}$ (liver; Müller et al. 2011). The possibility exists that such differences might contribute to predictive errors for metabolic rates in individuals $<10 \mathrm{~kg}$ (Figs. 6a and 8a). That possibility should not be overlooked, although it occurs within all intra-specific metabolic predictions that include both adolescent and mature individuals.

\section{A human allometric contribution}

The theoretical (Brody 1945; Heusner 1982a; SchmidtNielsen 1984; Darveau et al. 2002; White and Kearney 2014) and inter-specific experimental evidence (Sarrus and Rameaux 1838; Kleiber 1932; White and Seymour 2005) overwhelmingly supports using allometric analyses for scaling metabolic rate, whether those data were collected under basal (White and Seymour 2003) or exercising states (Nevill et al. 1992). In addition, we now also know that a universal, inter-specific scaling exponent does not exist (Heusner 1982a; White and Seymour 2003; Sieg et al. 2009), although that was once thought to be the case (Rubner 1883; mass ${ }^{0.67}$; Kleiber 1932; mass ${ }^{0.75}$ ). Instead, the mass exponent varies across species, ranging between mass ${ }^{0.5}$ and mass ${ }^{1.0}$, with its magnitude increasing with the inclusion of larger species (White et al. 2009; White and Kearney 2014). Within mammals, that species-dependency shows some evidence of an inverse relationship with body mass (e.g. mass ${ }^{0.97}$ [rats], mass $^{0.885}$ [dogs], mass ${ }^{0.60}$ [pigs], mass ${ }^{0.547}$ [humans]), which may be related to variations in the sizes of the metabolically active organs, relative to the overall body mass. It is perhaps not unreasonable to suggest that natural selection was also instrumental in that diversification, and the current authors favour a heat-loss mechanism to explain that relationship.

Within normothermic conditions, human deep-body temperatures are remarkably stable, and similar across individuals (Taylor et al. 2014). Those temperatures are most commonly higher than ambient conditions, as in this experiment, with the consequence being an inexorable and unidirectional heat exchange (loss). That thermal energy passes through the skin and respiratory membranes. Smaller individuals, regardless of gender, have a larger mass-specific surface area through which heat is lost (Notley et al. 2016, 2017). However, when heat loss is not required, metabolic heat production must match its dissipation rate so that a thermoneutral body temperature can be regulated. Smaller individuals tend to cool faster, due to their larger mass-specific surface area and the greater thermal inertia of larger individuals. Consequently, under basal conditions, metabolic rate varies allometrically with body mass, with progressively larger individuals displaying an exponentially smaller metabolic response per unit mass (Taylor and Gordon 2019). That is, heavier individuals require less mass-specific metabolic heat to regulate their body temperatures.

Embedded within these allometric observations, there was an apparent gender dependency (Fig. 8b and c), with a significantly lower scaling exponent for females (Eq. 2h; mass ${ }^{0.40}$ ) than males (Eq. $2 \mathrm{i}$; mass ${ }^{0.46}$ ) for body masses ranging from 40 to $96 \mathrm{~kg}$. It is beyond the scope of this research to speculate on either the reality of those observations or their mechanistic explanation. In the first instance, whilst every attempt was made to eliminate flawed data (Schofield 1985), and while the current authors endeavoured to eliminate individuals who were not physically mature, neither party had any capacity to influence either data collection precision or experimental errors. It is, therefore, assumed that the filtration processes were not flawless. Nevertheless, the observed gender difference is consistent with the literature (Garn et al. 1953; Arciero et al. 1993; Buchholz et al. 2001), and it would seem that difference widens with bodymass increments.

\section{Conclusion}

To the best of our knowledge, the current observations represent the first verification that human basal metabolic rate scales allometrically with body mass within a homogeneous sample of healthy, adult males with standardised physical characteristics. In line with existing evidence from other mammals, a unique scaling exponent was observed, with the resulting mass exponents being between mass ${ }^{0.50}$ (resting) and mass ${ }^{0.55}$ (basal). Finally, when healthy males (mass ${ }^{0.46}$ ) and females (mass ${ }^{0.40}$ ) from the historical database were compared over the adult age and body-mass ranges $(>18$ years and 40-96 kg), statistically significant differences in the mass exponents were observed. Collectively, these observations have significant physiological implications, and they are applicable to exercise and work physiologists, as well as to dieticians and those who prescribe metabolism-dependent pharmacological preparations.

Acknowledgements This project was supported by grants from the Defence Science and Technology Group (Australia). The opinions expressed in this paper are those of the authors and do not reflect the official policy or position of the Defence Science and Technology Group, or the Australian Government. HMB was supported by an International Postgraduate Tuition Award (University of Wollongong, Australia). NAST was supported during the writing of this manuscript by the Brain Pool Program through the National Research Foundation of Korea (NRF), funded by the Ministry of Science and Information, Communication and Technology (Grant number: 2019H1D3A2A01061171). Parts of this manuscript have been published in abstract form (Bowes et al. 2015). 
Author contributions These experiments were undertaken at the Centre for Medical and Exercise Physiology (University of Wollongong, Australia). NAST conceived, and NAST, CAB and HMB designed and planned this research. HMB ran the experiments and, with $\mathrm{CAB}$, was responsible for data collection and analysis. NAST assisted with data analysis and interpretation, and was responsible for the historical database and writing the manuscript. All authors read and approved the final version of the manuscript and its submission for publication, and agree to be accountable for this work, ensuring that questions related to the accuracy or integrity of any part of the work are appropriately investigated and resolved. All persons designated as authors qualify for authorship, and all those who qualify for authorship have been listed as authors.

\section{Compliance with ethical standards}

Conflict of interest There are no conflicts of interest.

\section{References}

Albrecht GH, Gelvin BR, Hartman SE (1993) Ratios as a size adjustment in morphometrics. Am J Phys Anthropol 91:441-468

Arciero PJ, Goran MI, Poehlman ET (1993) Resting metabolic rate is lower in women than in men. J Appl Physiol 75:2514-2520

Australian Bureau of Statistics (2012) 4364.0.55.001. Australian Health Survey: First Results, 2011-12. In: Commonwealth of Australia, Canberra. https://www.abs.gov.au/AUSSTATS/abs@.nsf/Looku p/4338.0Main+Features100032011-13?OpenDocument

Batterham AM, Jackson AS (2003) Validity of the allometric cascade model at submaximal and maximal metabolic rates in exercising men. Respir Physiol Neurobiol 135:103-106

Boothby WM, Sandiford I (1922) Summary of the basal metabolism data on 8,614 subjects with especial reference to the normal standards for the estimation of the basal metabolic rate. J Biol Chem 54:783-803

Bowes HM, Burdon CA, Taylor NAS (2015) The scaling of human basal metabolic rate in adult males. Proc Austr Physiol Soc 46:54P

Bowes HM, Burdon CA, Taylor NAS (2017) A contribution to understanding the impact of variations in body mass on fractionating the metabolic burden of military load carriage. J Sci Med Sports 20:S75-S76

Brody S (1945) Bioenergetics and growth: with special reference to the efficiency complex in domestic animals. Reinhold Publishing Corporation, New York

Buchholz AC, Rafii M, Pencharz PB (2001) Is resting metabolic rate different between men and women? Br J Nutr 86:641-646

Cole TJ, Henry CJK (2005) The Oxford Brookes basal metabolic rate database - a reanalysis. Public Health Nutr 8:1202-1212

Darveau CA, Suarez RK, Andrews RD, Hochachka PW (2002) Allometric cascade as a unifying principle of body mass effects on metabolism. Nature 417:166-170

Durbin J, Watson GS (1950) Testing for serial correlation in least squares regression: I. Biometrika. 37:409-428

Durbin J, Watson GS (1951) Testing for serial correlation in least squares regression: II. Biometrika. 38:159-177

Durnin JVGA (1959) The use of surface area and of body-weight as standards of reference in studies on human energy expenditure. Br J Nutr 13:68-71

Elia M (1992) Organ and tissue contribution to metabolic rate. In: Kinney JM, Tucker HN (eds) Energy metabolism: tissue determinants and cellular corollaries. Raven Press, New York, pp 61-80

Garn SM, Clark LC, Harper RV (1953) The sex difference in the basal metabolic rate. Child Dev 24:215-222
Gould SJ (1966) Allometry and size in ontegeny and phylogeny. Biol Rev Camb Philos Soc 41:587-640

Green SB (1991) How many subjects does it take to do a regression analysis. Multivar Behav Res 26:499-510

Hall C, Figueroa A, Fernhall B, Kanaley JA (2004) Energy expenditure of walking and running: comparison with prediction equations. Med Sci Sport Exerc 36:2128-2134

Harris JA, Benedict FG (1919) A biometric study of basal metabolism in man. In: Carnegie Institution of Washington, Washington. Publication number 279.

Heusner AA (1982a) Energy metabolism and body size. I. Is the 0.75 mass exponent of Kleiber's equations a statistical artifact? Respir Physiol 48:1-12

Heusner AA (1982b) Energy metabolism and body size. II. Dimensional analysis and energetic non-similarity. Respir Physiol 48:13-25

Heusner AA (1991) Body mass, maintenance and basal metabolism in dogs. J Nutr 121:S8-S17

Holliday MA (1971) Metabolic rate and organ size during growth from infancy to maturity and during late gestation and early infancy. Pediatrics 47:169-179

Holliday MA, Potter D, Jarrah A, Bearg S (1967) The relation of metabolic rate to body weight and organ size. Pediatr Res 1:185-195

Jetté M, Sidney K, Blümchen G (1990) Metabolic equivalents (METS) in exercise testing, exercise prescription, and evaluation of functional capacity. Clin Cardiol 13:555-565

Johnstone AM, Murison SD, Duncan JS, Rance KA, Speakman JR (2005) Factors influencing variation in basal metabolic rate include fat-free mass, fat mass, age, and circulating thyroxine but not sex, circulating leptin, or triiodothyronine. Am J Clin Nutr 82:941-948

Kim H-Y (2013) Statistical notes for clinical researchers: assessing normal distribution (2) using skewness and kurtosis. Restorat Dentistry Endodontics 38:52-54

Kleiber M (1932) Body size and metabolism. Hilgardia 6:315-353

Kleiber M (1950) Physiological meaning of regression equations. J Appl Physiol 2:417-423

Kleiber M (1961) The fire of life: an introduction to animal energetics. Wiley, London

Krogh A (1916) The respiratory exchange of animals and man. Longmans, Green and Company, London

Kvist A, Lindström $\AA$ (2001) Basal metabolic rate in migratory waders: intra-individual, intraspecific, interspecific and seasonal variation. Funct Ecol 15:465-473

Mansell PI, Macdonald IA (1990) Reappraisal of the Weir equation for calculation of metabolic rate. Am J Physiol 258:R1347-R1354

Markovic G, Vucetic V, Nevill AM (2007) Scaling behaviour of $\mathrm{O}_{2}$ in athletes and untrained individuals. Ann Hum Biol 34:315-328

McClave SA, Lowen CC, Kleber MJ, McConnell JW, Jung LY, Goldsmith LJ (2003) Clinical use of the respiratory quotient obtained from indirect calorimetry. J Parent Enteral Nutr 27:21-26

Motulsky HJ, Ransnas LA (1987) Fitting curves to data using nonlinear regression: a practical and nonmathematical review. FASEB J 1:365-374

Müller MJ, Langermann D, Gehrke I, Later W, Heller M, Glüer CC, Heymsfield SB, Bosy-Westphal A (2011) Effect of constitution on mass of individual organs and their association with metabolic rate in humans-a detailed view on allometric scaling. PLoS ONE 6:e22721

Müller MJ, Wang Z, Heymsfield SB, Schautz B, Bosy-Westphal A (2013) Advances in the understanding of specific metabolic rates of major organs and tissues in humans. Curr Opin Clin Nutr Metabolic Care 16:501-508

Müller MJ, Geisler C, Hübers M, Pourhassan M, Braun W, BosyWestphal A (2018) Normalizing resting energy expenditure across 
the life course in humans: challenges and hopes. Eur J Clin Nutr 72:628-637

Nevill AM, Ramsbottom R, Williams C (1992) Scaling physiological measurements for individuals of different body size. Eur J Appl Physiol 65:110-117

Noblet J, Fortune H, Shi XS, Dubois S (1994) Prediction of net energy value of feeds for growing pigs. J Anim Sci 72:344-354

Notley SR, Park J, Tagami K, Ohnishi N, Taylor NAS (2016) Morphological dependency of cutaneous blood flow and sweating during compensable heat stress when heat-loss requirements are matched across participants. J Appl Physiol 121:25-35

Notley SR, Park J, Tagami K, Ohnishi N, Taylor NAS (2017) Variations in body morphology explain sex differences in thermoeffector function during compensable heat stress. Exp Physiol 102:545-562

Owen OE, Holup JL, D’Alessio DA, Craig ES, Polansky M, Smalley KJ, Kavle EC, Bushman MC, Owen LR, Mozzoli MA, Kendrick ZV, Boden GH (1987) A reappraisal of the caloric requirements of men. Am J Clin Nutr 46:875-885

Packard GC, Boardman TJ (1988) The misuse of ratios, indices, and percentages in ecophysiological research. Physiol Zool 61:1-9

Packard GC, Boardman TJ (1999) The use of percentages and sizespecific indices to normalize physiological data for variation in body size: wasted time, wasted effort? Comp Biochem Physiol A: Mol Integr Physiol 122:37-44

Packard GC, Birchard GF, Boardman TJ (2011) Fitting statistical models in bivariate allometry. Biol Rev 86:549-563

Quenouille MH, Boyne AW, Fisher WB, Leitch I (1951) Statistical studies of recorded energy expenditure of man. In: Part I. Basal metabolism related to sex, stature, age, climate and race. Commonwealth Bureau of Animal Nutrition, Technical Communication No.17. Commonwealth Agricultural Bureau, Aberdeen, Scotland

Refinetti R (1989) Body size and metabolic rate in the laboratory rat. Exp Biol 48:291-294

Rogers DM, Olson BL, Wilmore JH (1995) Scaling for the $\mathrm{O}_{2}$-to-body size relationship among children and adults. J Appl Physiol 79:958-967

Ross WD, Marfell-Jones MJ (1991) Kinanthropometry. In: MacDougall JD, Wenger HA, Green HJ (eds) Physiological testing of the highperformance athlete. Human Kinetics, Champaign, pp 223-308

Ross WD, Wilson NC (1974) A strategem for proportional growth assessment. Acta Paediatr Belgica 28:169

Rubner M (1883) Ueber den Einfluss der Köpergrösse auf Stoff- und Kraftwechsel. Zeitschr für Biol 19:535-562

Sarrus F, Rameaux J (1838) Rapport sur un mémoire adressé à l'Académie Royale de Médecine. Bull l'Acad R Méd 3:1094-1100

Schmidt-Nielsen K (1984) Scaling: why is animal size so important?. Cambridge University Press, Cambridge

Schofield WN (1985) Predicting basal metabolic rate, new standards and review of previous work. Hum Nutr Clin Nutr 39:5-41
Shapiro SS, Wilk MB (1965) An analysis of variance test for normality (complete samples). Biometrika 52:591-611

Shephard RJ (2017) Open-circuit respirometry: a brief historical review of the use of Douglas bags and chemical analyzers. Eur J Appl Physiol 117:381-387

Sholl D (1948) The quantitative investigation of the vertebrate brain and the applicability of allometric formulae to its study. Proc R Soc Lond B 135:243-258

Sieg AE, O'Connor MP, McNair JN, Grant BW, Agosta SJ, Dunham AE (2009) Mammalian metabolic allometry: do intraspecific variation, phylogeny, and regression models matter? Am Nat 174:720-733

Tanner JM (1949) Fallacy of per-weight and per-surface area standards, and their relation to spurious correlation. J Appl Physiol 2:1-15

Taylor NAS, Gordon CJ (2019) The origin, significance and plasticity of the thermoeffector thresholds: extrapolation between humans and laboratory rodents. J Therm Biol 85:102397

Taylor NAS, Tipton MJ, Kenny GP (2014) Considerations for the measurement of core, skin and mean body temperatures. J Therm Biol 46:72-101

Taylor NAS, Shephard RJ, Lindinger MI (2018) Foundational insights into the estimation of whole-body metabolic rate. Eur J Appl Physiol 118:867-874

Todd G, Gordon CJ, Groeller H, Taylor NAS (2014) Does intramuscular thermal feedback modulate eccrine sweating in exercising humans? Acta Physiol 212:86-96

Weir JBDV (1949) New methods for calculating metabolic rate with special reference to protein metabolism. J Physiol 109:1-9

White CR, Kearney MR (2014) Metabolic scaling in animals: methods, empirical results, and theoretical explanations. Comprehen Physiol 4:231-256

White CR, Seymour RS (2003) Mammalian basal metabolic rate is proportional to body mass ${ }^{2 / 3}$. Proc Natl Acad Sci USA 100:4046-4049

White CR, Seymour RS (2005) Sample size and mass range effects on the allometric exponent of basal metabolic rate. Comp Biochem Physiol A Mol Integr Physiol 142:74-78

White CR, Blackburn TM, Seymour RS (2009) Phylogenetically informed analysis of the allometry of mammalian basal metabolic rate supports neither geometric nor quarter power scaling. Evol Int J Org Evol 63:2658-2667

White CR, Frappell PB, Chown SL (2012) An information-theoretic approach to evaluating the size and temperature dependence of metabolic rate. Proc R Soc Lond B 279:3616-3621

Xiao X, White EP, Hooten MB, Durham SL (2011) On the use of log transformation vs. nonlinear regression for analyzing biological power laws. Ecology 92:1887-1894

Publisher's Note Springer Nature remains neutral with regard to jurisdictional claims in published maps and institutional affiliations. 